\title{
K-band Extragalactic Background Light from Deep Galaxy Counts in the Subaru Deep Field
}

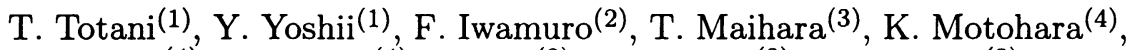

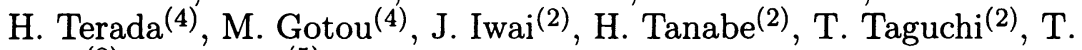
$\mathrm{Hata}^{(2)}, \& \mathrm{~S}$. Oya ${ }^{(5)}$

(1) Institute of Astronomy, School of Science, University of Tokyo, Mitaka, Tokyo 181-8588, Japan

(2) Department of Physics, Kyoto University, Kitashirakawa, Kyoto 606-8502, Japan

(3) Department of Astronomy, Kyoto University, Kitashirakawa, Kyoto 606-8502, Japan

(4) Subaru Telescope, National Astronomical Observatory, $650 \mathrm{~N}$ Aohoku Place, Hilo, HI 96720, USA

(5) Communications Research Laboratory, Koganei, Tokyo 184-8975, Japan

\begin{abstract}
We report estimates for the extragalactic background light (EBL) in the $K$ band $(2.2 \mu \mathrm{m})$, obtained by the integration of galaxy counts down to $K=25 \mathrm{mag}$ in the Subaru Deep Field (SDF, $2^{\prime} \times 2^{\prime}$ ). We have obtained deep galaxy count data by using the $8.2 \mathrm{~m}$ Subaru telescope, with a total integration time of 10 hours and an average seeing of about 0.4 arcsec. The 5 -sigma limiting magnitude is $K=23.5$, and 350 objects are detected brighter than this magnitude. There has been a significant discrepancy between previous $K$-count observations, probably because of the systematic uncertainties in the completeness correction. To overcome this problem, we have paid special attention to selection effects and completeness corrections, with realistic theoretical galaxy models taken into account consistently. The faint-end slope is significantly flatter than some earlier observations of $K$ counts, and our results suggest that the bulk of the extragalactic light in this band has been resolved as discrete sources. We estimate the value of the EBL flux obtained from the integration of our counts as $9.8 \pm 1.0 \mathrm{nW} \mathrm{m}^{-2} \mathrm{sr}^{-1}$.
\end{abstract}

